\title{
BMJ Open Detection of IgM, IgG and SARS-CoV-2 RNA among the personnel of the University of Milan, March through May 2020: the UNICORN study
}

\author{
Gregorio Paolo Milani, ${ }^{1}$ Federica Rota, ${ }^{1}$ Chiara Favero, ${ }^{1}$ Laura Dioni, ${ }^{1}$ \\ Alessandro Manenti, ${ }^{2}$ Mirjam Hoxha, ${ }^{1}$ Elena Pariani, ${ }^{3}$ Benedetta Albetti, ${ }^{1}$ \\ Angela Cecilia Pesatori, ${ }^{1}$ Emanuele Montomoli, ${ }^{4}$ Valentina Bollati (D) , ${ }^{1}$ on behalf of \\ the UNICORN Consortium investigators
}

To cite: Milani GP, Rota F, Favero C, et al. Detection of $\operatorname{lgM}, \lg \mathrm{G}$ and SARS-CoV-2 RNA among the personnel of the University of Milan, March through May 2020: the UNICORN study. BMJ Open 2021;11:e046800. doi:10.1136/ bmjopen-2020-046800

- Prepublication history for this paper is available online. To view these files, please visit the journal online (http://dx.doi. org/10.1136/bmjopen-2020046800).

Received 09 November 2020 Revised 03 March 2021 Accepted 09 March 2021

Check for updates

(c) Author(s) (or their employer(s)) 2021. Re-use permitted under CC BY-NC. No commercial re-use. See rights and permissions. Published by BMJ.

${ }^{1}$ Department of Clinical Sciences and Community Health,

University of Milan, Milano, Italy

${ }^{2}$ VisMederi Research, Milan, Italy

${ }^{3}$ Department of Biomedical

Sciences for Health, University

of Milan, Milano, Italy

${ }^{4}$ Department of Molecular and Developmental Medicine, University of Siena, Siena, Italy

Correspondence to

Professor Valentina Bollati;

valentina.bollati@unimi.it

\section{ABSTRACT}

Objectives In Italy, the pandemic of COVID-19 resulted in congestion of hospitals and laboratories and probably determined an underestimation of the number of infected subjects, as the molecular diagnosis of SARS-CoV-2 infection was mainly performed on hospitalised patients. Therefore, limited data are available about the number of asymptomatic/paucisymptomatic subjects in the general population across time. To understand SARS-CoV-2 infection in the general population, we have developed a cross-sectional study (the 'UNIversity against CORoNavirus study') to investigate infection trends in asymptomatic/ paucisymptomatic subjects in Milan (Italy), between March and June 2020

Participants The study population included 2023 subjects asymptomatic at the enrolment.

Primary outcome measures A nasal mid-turbinate swab for the detection of SARS-CoV-2 RNA and blood specimen for testing serum antibodies (immunoglobulin M (IgM) and $\mathrm{lgG}$ ) were collected.

Results Subjects showing positivity for the SARS-CoV-2 RNA and/or for anti-SARS-CoV-2 lg is $237(11.7 \%)$. Only $1.2 \%(n=25)$ of the total population had a positive nasal swab for SARS-CoV-2 and the large majority (21/25) of them were observed in March. A total of 226 subjects $(11 \%)$ had $\lg M(n=19 ; 0.9 \%)$, IgG $(n=155 ; 7.7 \%)$ or both $(n=52 ; 2.6 \%)$ against SARS-CoV-2. Subjects with a present or past SARS-CoV-2 infection did not differ from other subjects as regards the number of cohabiting family members, travels, fever and upper and lower respiratory infection episodes.

Conclusions Results from the present study support the hypothesis that the actual spread of the virus in Lombardy was underestimated in the official records. However, as it is not known how long Ig persist, numbers should be taken cautiously.

\section{INTRODUCTION}

After the COVID-19 outbreak in China in January 2020, the European countries have been the first to be affected by this viral emergency during the following months. ${ }^{1}$ In Italy,

\section{Strengths and limitations of this study}

- A strength of this study is that all the parts of the UNIversity against CORoNavirus study project have been conducted in different phases of the pandemic, allowing us to investigate the trend occurring across the months.

- The recruitment was dedicated to subjects working at the University of Milan, Italy, limiting the possibility to extend our findings to the general population.

- If it is easy to depict a timeframe for symptomatic subjects (as they reach the hospital/doctor as soon as the symptoms develop), the study of asymptomatic subjects does not allow to delineate precisely how long before the subject became infected.

the first death attributable to SARS-CoV-2 infection was observed on 21 February 2020, and was followed by a rapid viral spread, resulting in 110574 documented cases and 13155 documented deaths related to SARS-CoV-2 infection as of 1 April 2020.

In Italy, the unexpected pandemic of COVID-19 resulted in congestion of both hospitals and laboratories and led to focusing on symptomatic and hospitalised patients ${ }^{23}$ for whom the molecular diagnosis of SARS-CoV-2 infection was mainly performed. Although each country has adopted different measures to contrast SARS-CoV-2 diffusion, the main strategy applied by most was the lockdown. ${ }^{4}$ Italy has adopted a national lockdown from 9 March to 3 May 2020.

Limited data are available about the number of infected people who are asymptomatic or paucisymptomatic across time in the general population. ${ }^{5}$ However, it is now recognised that most subjects infected by the SARS-CoV-2 do not develop symptoms or present only mild clinical findings, ${ }^{6}$ making 
the traceability of these subjects of paramount importance due to the possibility that they act as virus spreader. ${ }^{7}$

To obtain a better understanding of SARS-CoV-2 infection in the general population, we implemented a crosssectional study (the 'UNIversity against CORoNavirus study', UNICORN) to investigate the trend of infections among asymptomatic or paucisymptomatic subjects in the area of Milan (Lombardy, Italy), one of the cities with the major burden of SARS-CoV-2 infection in Europe. The study was developed in two phases: the first phase regards 197 subjects recruited on 30 and 31 March 2020 (lockdown); the second phase was implemented from 13 May to 23 June 2020 (reopening of activities) and involved 1.826 subjects. In both phases, a nasal swab and a blood sample were collected from each study participant.

The main objective of this study was to provide a snapshot of the actual spread of the SARS-CoV-2 virus among asymptomatic or paucisymptomatic subjects across time. The secondary objective involved the characterisation of the subjects who get the infection and the evaluation of the seroprevalence trend of immunoglobulins (Ig) against SARS-CoV-2 in the study population.

\section{METHODS}

\section{Inclusion criteria and study procedures}

For the UNICORN study, eligible were all the personnel of the University of Milan, which include a total of 3973 employers. Exclusion criteria included: subjects with fever, any symptoms of influenza-like infections or dyspnoea at the moment of the recruitment or in the 14 previous days, subjects with close and prolonged contact with any person positive for SARS-CoV-2 or with signs or symptoms suggestive for infection in the previous 14 days (eg, a household member with respiratory symptoms or fever). The personnel received the study invitation through their institutional email address. After registering on an institutional web page dedicated to the UNICORN study, subjects received written instructions on study procedures to avoid any possible source of safety risk. An appointment at an arranged time was set for each participant at one of the two university campuses that were fully dedicated to the study. Once at the campus, participants had to confirm that they did not present any of the conditions listed in the exclusion criteria, and dedicated personnel measured twice their body temperature by an infrared thermometer. If the mean body temperature was $<37.5^{\circ} \mathrm{C}$, participants could undergo specimen sampling collection. The day after, all participants filled in an anonymous online questionnaire.

\section{Specimen sampling}

A nasal mid-turbinate swab (DID Diagnostic International, Milan, Italy) was collected through a supervised onsite self-collection according to the Centers for Disease Control and Prevention Guideline for 'Collecting, Handling, and Testing Clinical Specimens from Persons for Coronavirus Disease 2019 (COVID-19)'. The swab was then immediately placed in a tube of Universal Transport Media (DID Diagnostic International) and frozen at $-80^{\circ} \mathrm{C}$ until RNA extraction. After nasal swab collection, a venous blood sample $(7.5 \mathrm{~mL})$ was drawn in EDTA tubes, following standard procedures. Each blood sample was processed within 4 hours to obtain the plasma fraction.

\section{Laboratory analyses}

Nasal mid-turbinate swab

The QIAamp Viral RNA Mini Kit (Qiagen, Hilden, Germany) was used for RNA isolation in swabs, according to the manufacturer's instructions. Before RNA extraction, an aliquot of $140 \mu \mathrm{L}$ of the viral transport medium was inactivated in lysis buffer (Buffer AVL, Qiagen; $560 \mu \mathrm{L}$ ). In each sample, $10 \mu \mathrm{L}$ of internal control RNA (MS2 Phage Control included in TaqPath COVID-19 Kit) and an RNA carrier were added. The purified RNA was eluted in $50 \mu \mathrm{L}$ and immediately stored at $-80^{\circ} \mathrm{C}$.

To detect SARS-CoV-2 RNA, a multiplex real-time RT-PCR test (TaqPath COVID-19 CE-IVD RT-PCR Kit, Thermo Fisher Scientific) was applied. ${ }^{8}$ Five microlitres of RNA was reverse transcribed into cDNA and amplified using the QuantStudio 12K Flex Real-Time PCR Instruments (Applied Biosystems, California, USA). The reaction was run in a 384-well plate prepared by a Microlab Starlet robot (Hamilton Robotics, Bonaduz, Switzerland).

In the PCR reaction, the probes anneal to three specific SARS-CoV-2 sequences: (1) ORFlab with reporter dye FAM; (2) N protein (nucleocapsid) with reporter dye VIC; and (3) S protein (spike) with reporter dye ABY. In addition, a bacteriophage MS2 Control (with reporter dye JUN) is also amplified to verify the efficacy of the sample preparation and the absence of inhibitors. The reaction mix added to each RNA sample $(5 \mu \mathrm{L})$ is the following: $5 \mu \mathrm{L}$ of TaqPath 1-Step Multiplex Master Mix (No ROX) (4X), $1 \mu \mathrm{L}$ of TaqPath COVID-19 Assay Multiplex and 9 $\mu \mathrm{L}$ nuclease-free water.

In each run, a standard curve was added to check the efficiency of the amplification; the standard TaqPath COVID-19 Control $(1 \times 104$ copies $/ \mu \mathrm{L})$ was diluted serially in TaqPath COVID-19 Control Dilution Buffer 1/4fold per dilution to produce six concentrations of copies, ranging from 312.5 to 0.305 copies $/ \mu \mathrm{L}$. Five microlitres of each standard was then distributed in the 'standard curve' 1000 wells. The plates were then sealed, centrifuged briefly at $800 \times g$ and located in QuantStudio $12 \mathrm{~K}$ Flex Real-Time PCR Instruments (Applied Biosystems) for the run. The passive reference was set to 'None', and ‘Absolute Quantification' as run type.

The following thermal protocol was applied: $2 \mathrm{~min}$ at $25^{\circ} \mathrm{C}$ for UNG (Uracil-DNA glycosylase) activation, 10 $\min$ at $53^{\circ} \mathrm{C}$ for the reverse transcription reaction, $2 \mathrm{~min}$ at $95^{\circ} \mathrm{C}$ for activation, 40 cycles of denaturation at $95^{\circ} \mathrm{C}$ for $3 \mathrm{~s}$ and anneal/extension at $60^{\circ} \mathrm{C}$ for $30 \mathrm{~s}$.

The data analysis was performed using the 'Design and Analysis Software' (V.2.3.3, Applied Biosystems) setting 'Automatic Threshold'. The reaction was considered only if MS2 Ct $\leq 38$. If any two of the three SARS-CoV-2 genes 
were positive $(\mathrm{Ct} \leq 38)$ the sample was classified as positive; if only one of the assays was positive, the test was repeated. Once the test was repeated, and the result was again positive, the sample was classified positive for SARS-CoV-2. If all three of the assays were negative ( $\mathrm{Ct}=$ undetermined), the subject was classified as negative.

\section{Blood analyses}

Blood EDTA was centrifuged at $1200 \mathrm{~g}$ for $15 \mathrm{~min}$ to obtain cell-free plasma. Immunoglobulin $\mathrm{M}(\operatorname{IgM})$ and IgG against SARS-CoV-2 in plasma samples were tested.

The Wantai anti-SARS-CoV-2 IgM ELISA (Beijing Wantai Biological Pharmacy Enterprise, Beijing, China) ${ }^{9}$ was performed according to the manufacturer's instructions. Reported sensitivity is $86 \%$ and specificity is $100 \%$. The assays detect antibodies binding SARS-CoV-2 spike protein receptor-binding domain (RBD) in human serum or plasma. Briefly, $10 \mu \mathrm{L}$ plasma samples and 100 $\mu \mathrm{L}$ of specimen diluent were added to wells coated with antibodies directed against the human IgM proteins and incubated for $30 \mathrm{~min}$ at $37^{\circ} \mathrm{C}$. Each well was aspirated and washed five times using an automatic microplate washer (MicroFill Dispenser, BioTek Instruments, Winooski, Vermont, USA). Then, a recombinant horseradish peroxidase (HRP)-conjugated SARS-CoV-2 antigen was added and incubated for $30 \mathrm{~min}$ at $37^{\circ} \mathrm{C}$. After further washing, a chromogen solution was added. The reaction was stopped after $15 \mathrm{~min}$ at $37^{\circ} \mathrm{C}$, and the resultant absorbance was read on a microplate reader (Synergy HT, BioTek Instruments) at $450 \mathrm{~nm}$ with reference at $620 \mathrm{~nm}$. The cut-off value for a positive result was calculated according to the manufacturer's instruction and equal to 0.105 for antiSARS-CoV-2 IgM ELISA.

To perform RBD ELISA IgG, ELISA plates were coated with $1 \mu \mathrm{g} / \mathrm{mL}$ of purified recombinant spike-RBD HEKderived protein (Sino Biological, Beijing, China); the assay was set and qualified as already reported. ${ }^{10}$ After overnight incubation at $+4^{\circ} \mathrm{C}$, coated plates were washed three times with $300 \mu \mathrm{L} /$ well of ELISA washing solution containing Tris-buffered saline (TBS)-0.05\% Tween 20, then blocked for 1 hour at $37^{\circ} \mathrm{C}$ with a solution of TBS containing $5 \%$ of non-fat dry milk (Euroclone, Pero, Italy).

Human serum samples were heat inactivated at $56^{\circ} \mathrm{C}$ for 1 hour to reduce the risk of the presence of intact and infectious viruses in the sample, then diluted 1:100 in TBS- $0.05 \%$ Tween $205 \%$. Plates were washed three times as previously then $100 \mu \mathrm{L}$ of each serum dilution was added to the coated plates and incubated for 1 hour at $37^{\circ} \mathrm{C}$. Next, after the washing step, $100 \mu \mathrm{L} /$ well of Goat anti-Human IgG-Fc HRP-conjugated antibody diluted 1:100.000 (Bethyl Laboratories, Montgomery, Texas, USA) was added. Plates were incubated at $37^{\circ} \mathrm{C}$ for 30 min. Following incubation, the plates were washed and $100 \mu \mathrm{L} /$ well of $3,3^{\prime}, 5,5^{\prime}$-tetramethylbenzidine substrate (Bethyl Laboratories) was added and incubated in the dark at room temperature for $20 \mathrm{~min}$. The reaction was stopped by adding $100 \mu \mathrm{L}$ of ELISA stop solution (Bethyl
Laboratories) and read at $450 \mathrm{~nm}$. The cut-off value was established as three times the average of optical density (OD) values from blank wells (background-no addition of analyte). Samples with the ODs under the cut-off value at the first (1:100) dilution were assigned as negative; samples where the ODs at 1:100 dilution were above the cut-off value were assigned as positive. Borderline samples were defined where one replicate was under the cut-off and the other was above. The reported sensitivity of this method is $85.7 \%$ and specificity is $98.1 \%$.

\section{Questionnaire}

An online structured questionnaire was filled in by the enrolled subjects to collect the following information: age, gender, body height and weight, education level, smoking, number of cohabitants (and the number of cohabitants aged 10 years or less), residence area, means of transport and time for commuting to work, lifestyle, influenza vaccination in the last year, underlying chronic diseases and treatments, and travels to Europe, North America, South America, Oceania, Africa and Asia from 1 October 2019. Also, episodes of fever, upper and lower respiratory infections (including their duration) from 1 October 2019, medical comorbidities and treatments were investigated.

\section{Statistical methods}

To have a homogenous distribution of subject analyses during the second phase (13 May to 23 June 2020), a total of 250-300 subjects were investigated each week. The percentage of subjects with a positive nasal swab for SARS-CoV-2 and with IgM and IgG against the virus was calculated for each week of the study project. Categorical data were reported as absolute numbers and frequencies and the quantitative variables are expressed as the mean \pm SD. To investigate the characteristics of study participants who had been infected by the SARS-CoV-2 (ie, with a positive nasal swab or with at least IgM or IgG against SARS-CoV-2), the Pearson $\chi^{2}$ test or Fisher's exact test for categorical variables and t-test for continuous variables were used. We also computed the OR and 95\% CIs to evaluate the associations of demographical and clinical characteristics of subjects who had been infected by the SARS-CoV-2 using logistic regression models. Statistical analyses and graphs were performed with SAS software (V.9.4; SAS Institute).

\section{RESULTS}

A total of 2111 subjects (53\% of the University of Milan employers) registered for the present study. Eightyeight subjects $(4.2 \%)$ revoked their participation before sample collection or before completing the questionnaire. The main characteristics of 2023 enrolled subjects are described in table 1.

The total number of subjects resulting to be positive to at least one of the SARS-CoV-2 tests (nasal swab and/ or serological test) is $237(11.7 \%)$. Only $1.2 \%(\mathrm{n}=25)$ of 
Table 1 Characteristics of study participants with a present or past SARS-CoV-2 infection

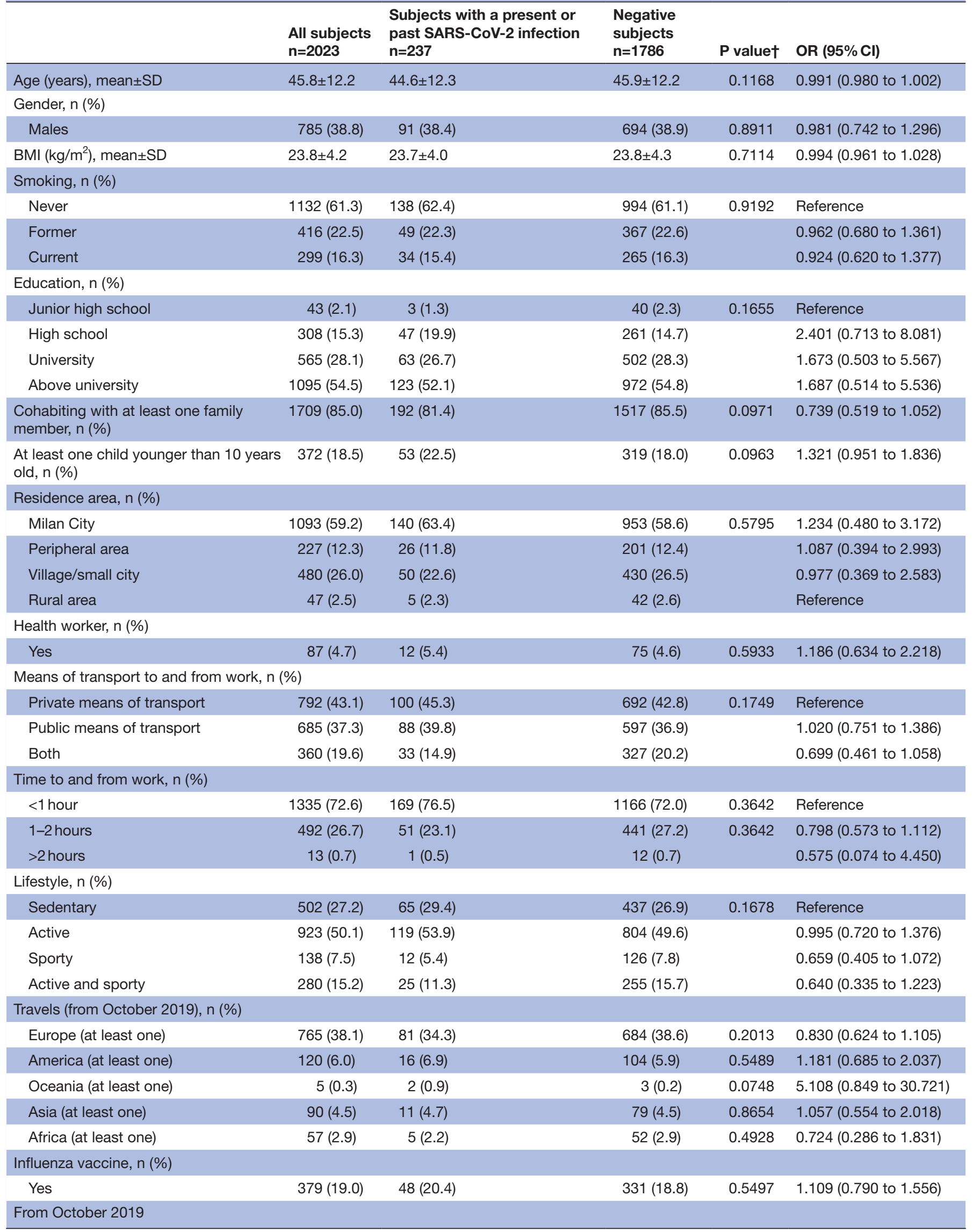


Table 1 Continued

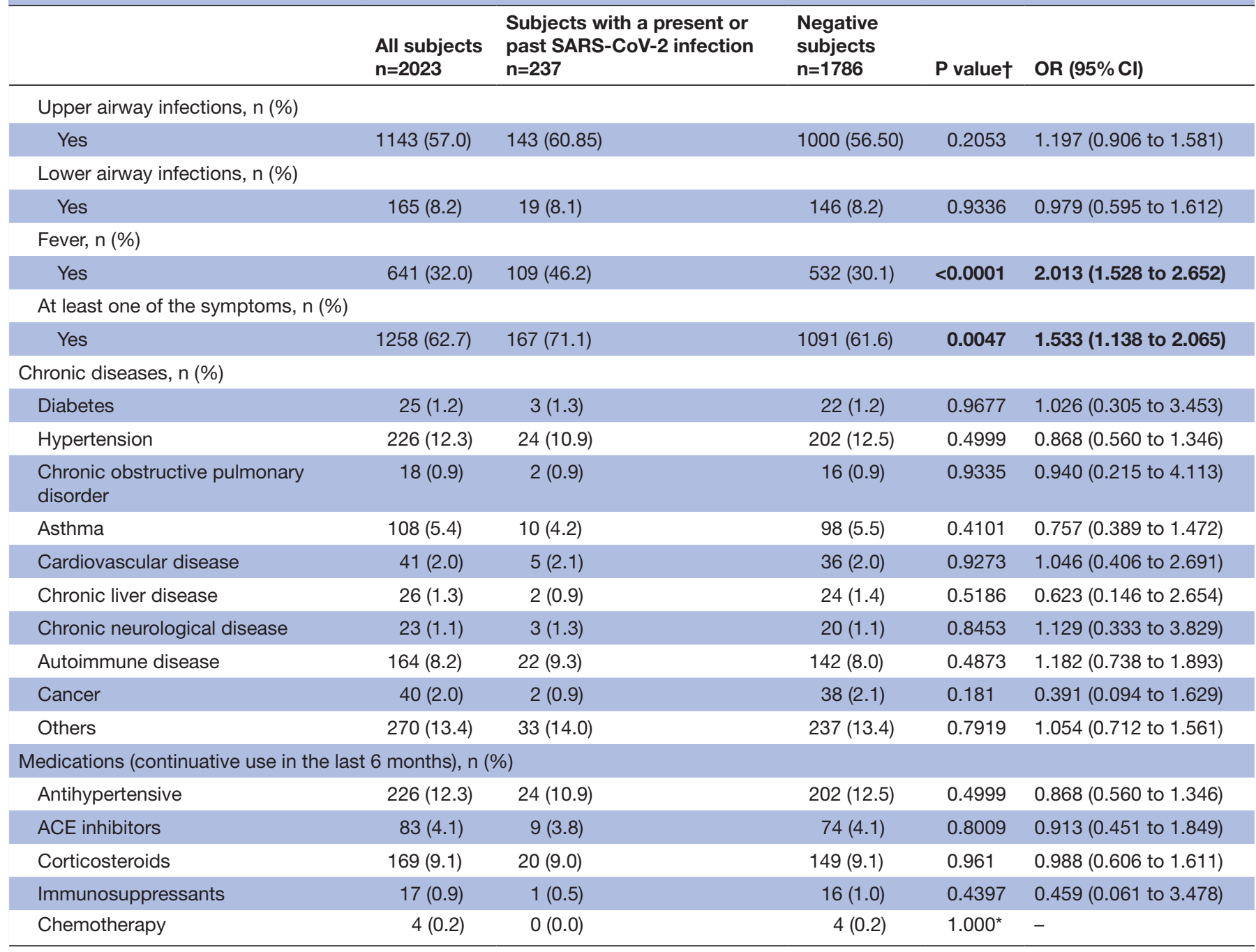

Continuous variables are expressed as mean \pm SD, discrete variables are expressed as counts (\%).

The $p$ values were calculated by $\chi^{2}$ test.

${ }^{*}$ Fisher's exact test.

†P values from t-test. $p$-value $<0.05$ are reported in bold.

BMI, body mass index.

the total population had a positive nasal swab, whereas 226 subjects $(10.7 \%)$ had antibodies against SARS-CoV-2: $19(0.9 \%)$ presented only IgM, $155(7.7 \%)$ only IgG and $52(2.6 \%)$ both IgM and IgG. The co-occurrence of the different markers is described in figure 1. Eighty-eight per cent of the study population was negative to all the SARS-CoV-2 tests. About $6 \%$ showed only IgG and $2 \%$ had both $\operatorname{IgG}$ and $\operatorname{IgM}$. A very small number of subjects are included in each of the other possible test combinations.

Seventy-one per cent of subjects with a present or past SARS-CoV-2 infection reported at least one of the symptoms potentially attributable to SARS-CoV-2 infection occurring from October 2019 to the 2 weeks before recruitment (table 1 ). The risk to have been infected by SARS-CoV-2 was doubled (OR 2.01; $95 \%$ CI 1.53 to 2.65) for subjects who had a fever and increased by about $50 \%$ (OR 1.53; 95\% CI 1.14 to 2.07) for subjects who presented at least one symptom (upper/lower airway infection or fever). About $30 \%$ of subjects with a present or past SARS-CoV-2 infection were fully asymptomatic. The risk to be infected did not vary across the other examined variables as shown in table 1 .

Figure 2 shows the trend of positive nasal swabs, IgM and IgG against SARS-CoV-2 during the whole enrolment period. The large majority $(21 / 25)$ of subjects with a positive nasal swab were observed in March. In detail, the prevalence of positive nasal swab was $11 \%$ $(21 / 197)$ in the first phase and $0.2 \%(4 / 1826)$ in the second phase.

The percentage of subjects positive for IgM did not vary across the whole study period, whereas the highest percentage of IgG-positive subjects was observed 4 weeks after the end of the lockdown measures $(n=38 ; 14.5 \%)$. A further slight increase was measured after 7 weeks $(n=29$; $10.6 \%)$. 


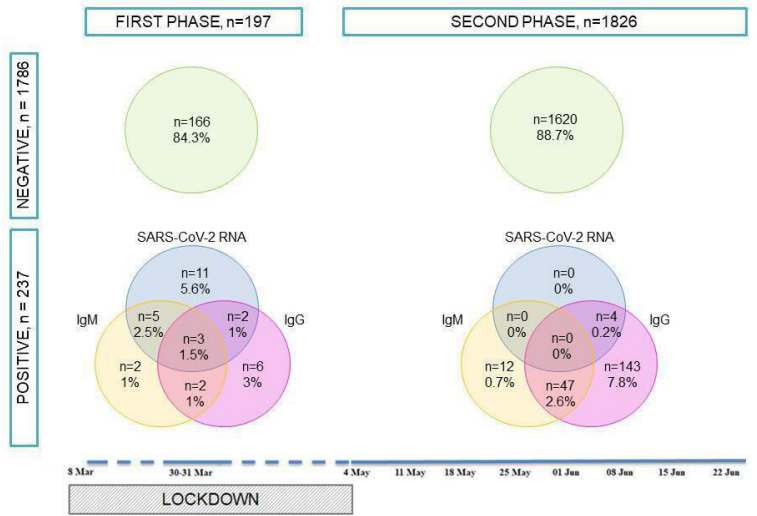

Figure 1 Venn diagram showing the number of subjects testing positive for SARS-CoV-2 RNA in the nasal swab (blue circles), for circulating IgM (yellow circles), IgG (violet circles) or negative for any markers (green circles). In the lower part of the figure, a timeline representing the study periods is also reported.

\section{DISCUSSION}

Among asymptomatic or paucisymptomatic subjects enrolled in the UNICORN project, the percentage of positive swabs changed across the study period. In particular, it was $11 \%$ among subjects enrolled in March 2020, while the lockdown was active, and declined to $0.2 \%$ among subjects enrolled in the following months (May to June 2020). These findings indicate a drastic decrease in virus spread among the population after the first epidemic wave. Recent data including a high number of countries around the world suggest that countries implementing lockdown measures presented a decreasing number of new patients with COVID-19 as compared with those that did not. ${ }^{11}$ The lockdown measures were also associated with a lower rate of COVID-19 deaths. ${ }^{12} 13$ Our data seem to support this hypothesis, even though the fact that the measures adopted during the lockdown have contributed to greatly slow down the spread of the virus and have contributed to interrupt the chain of infections among the population is under debate. ${ }^{14}$ Indeed, we cannot consider the lockdown, personal protective equipment and social distancing as the only factors able to contain the spread of the virus. ${ }^{9}$ For example, the spread and virulence of many types of viruses are strictly dependent on seasons, temperatures, humidity, ${ }^{15}$ air pollutant
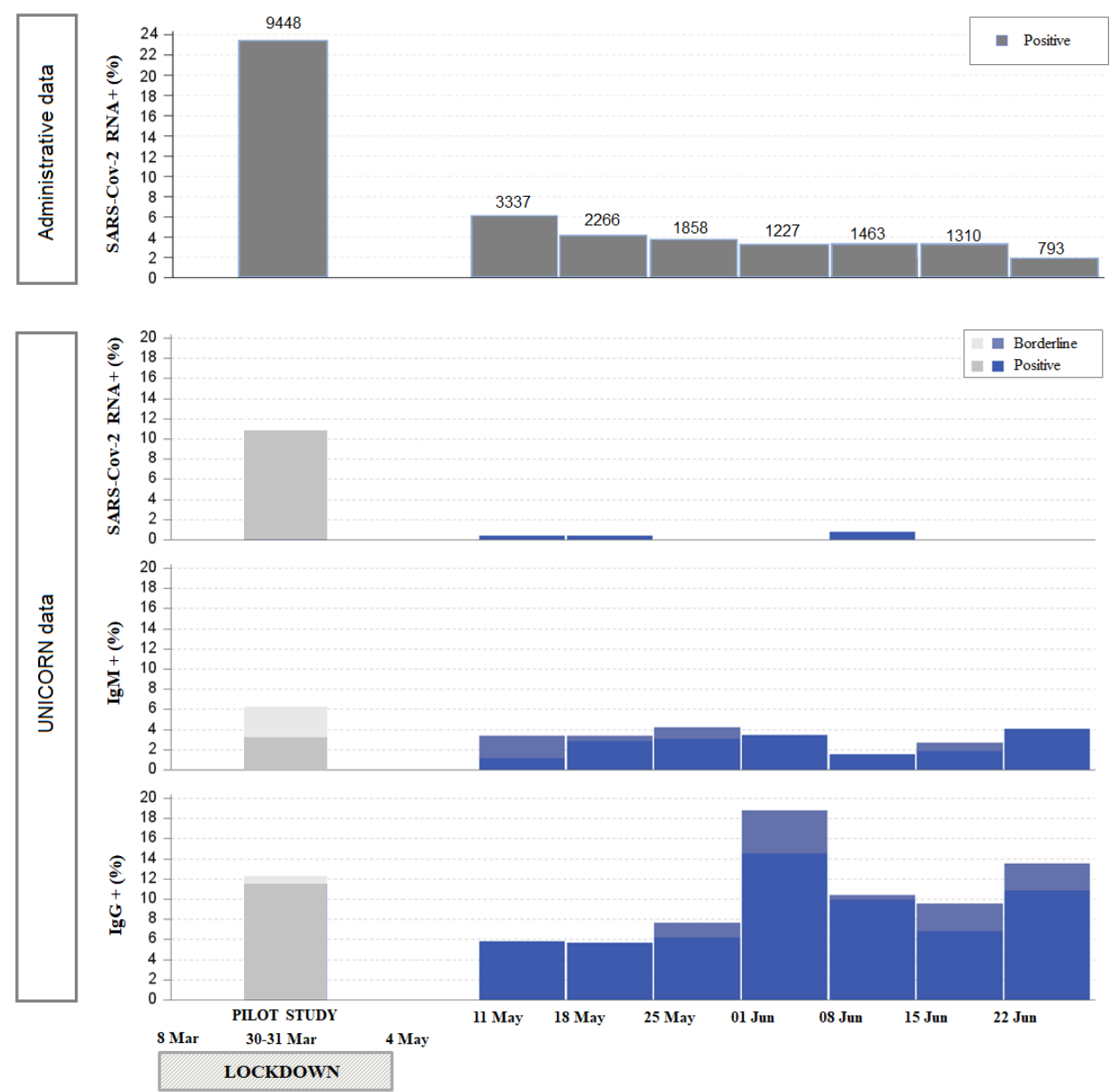

Figure 2 The upper panel shows the numbers and percentages of positive nasal swabs registered in the Lombardy region and reported by the Italian Ministry of Health (Ministero della Salute, http://www.salute.gov.it/portale/nuovocoronavirus/ homeNuovoCoronavirus.jsp). The lower panel shows the trends of positive nasal swabs, IgM and IgG against SARS-CoV-2 recorded during the whole enrolment period of the UNIversity against CORoNavirus study (UNICORN). 
exposure $^{16}$ or individual composition of microbiota as a susceptibility factor. ${ }^{17}$ Therefore, several factors may have contributed synergistically to the slowing of SARS-CoV-2 infections.

Although it is controversial if asymptomatic carriers of SARS-CoV-2 might be a means of infection for other subjects, ${ }^{5}$ many data suggest a possible role for them in the silent spread of the virus. ${ }^{18}$

The time window to define a subject as 'asymptomatic' instead of 'presymptomatic' is still not clearly defined and represents an additional challenge in COVID-19 research. However, data from longitudinal cohorts suggest that a minority of asymptomatic subjects testing positive for SARS-CoV-2 eventually develop symptoms. ${ }^{5}$

In addition, only ad hoc questions allow identifying underestimated symptoms. In our study population, for example, about $70 \%$ of subjects with a present or past SARS-CoV-2 infection showed at least one symptom in the months before recruitment, with an almost doubled risk to be infected for subjects with fever or mild respiratory symptoms.

It might be surprising that healthcare workers did not test positive for SARS-CoV-2 more frequently than other subjects, since they are considered at high risk of infection. ${ }^{19}$ However, according to the eligibility criteria of the study, we enrol only subjects who had no direct contact with patients potentially affected by the SARS-CoV-2.

The results of this study are partially in line with those obtained in a previous cross-sectional study performed on 5 May 2020 in Bergamo (Lombardy). ${ }^{20}$ On the other hand, a novel finding observed in this study is that we did not observe a clear trend in the increase of subjects with Ig against SARS-CoV-2 during the study period. These data might partially confirm that in Lombardy the spread of the virus has been limited during spring. On the other hand, a few studies found that the persistence of Ig against SARS-CoV-2 in asymptomatic carriers of SARS-CoV-2 might be shorter than one in symptomatic subjects. ${ }^{21}$ Accordingly, the longitudinal evaluation of seroprevalence of subjects from the UNICORN study showed that clearance of Ig occurs in approximately twothirds of subjects after 2 months ${ }^{22}$ and might explain the findings observed in this study.

A study conducted in several Chinese regions including more than 60000 asymptomatic subjects found that seroprevalence against SARS-CoV-2 was higher in females and older individuals. However, due to the retrospective design of this study, the authors could not evaluate the clinical history of the enrolled subjects. ${ }^{23}$ This study showed that a clinical history of symptoms possibly associated with a viral infection markedly increased the odds to have a laboratory test consistent with a SARS-CoV-2 infection. In particular, a history of fever in the previous months was associated with the highest risk of infection by SARS-CoV-2. This finding is consistent with the results of a recent systematic review showing that fever is the most frequent clinical manifestation in subjects infected by SARS-CoV-2. ${ }^{24}$
This study has some limitations. First, the recruitment was dedicated to subjects working at the University of Milan, Italy, limiting the possibility of the extent of our findings to the general population. However, the subjects recruited carry out different tasks and live throughout the Lombardy region, guaranteeing a good degree of heterogeneity of the population under study. Second, if it is easy to depict a timeframe for symptomatic subjects (as they reach the hospital/doctor as soon as the symptoms develop), the study of asymptomatic subjects does not allow to delineate precisely how long before the subject became infected. Although we are aware of this limit, the UNICORN population offers a unique opportunity to investigate asymptomatic subjects in an unbiased manner. Moreover, nasal samples were self-collected and this procedure might be fraught with inaccurate sampling. However, recent data point out that the mid-turbinate sample collection by the patients has a similar accuracy to the nasopharyngeal sample collection by healthcare personnel for the detection of SARS-CoV-2. ${ }^{25}$ A further limitation is the lack of any evaluation of neutralising antibodies and their relationship with immunoassay results. However, a satisfactory agreement with the immunoassay used in our study has been already reported. ${ }^{10}$ Finally, we did not measure circulating IgA that could be part of the humoral response to SARS-CoV-2 infection. ${ }^{26}$ A strength of this study is that all the parts of the UNICORN project have been conducted in different phases of the pandemic, allowing us to investigate the trend occurring across the months.

In conclusion, the results achieved gave us an insight into the spread of the virus that goes far beyond the track of the hospitalised symptomatic subjects. This study showed that subjects with fever or with an airway infection in previous months have a significantly higher risk to have been infected by the SARS-CoV-2 than subjects who had no such conditions. One of the main questions that remain open regards how long anti-SARS-CoV-2 antibodies remain in the body. In this context, the future perspectives of the UNICORN study include the monitoring of all the subjects showing a positive nasal swab or serological test, to assess their future risk of developing a further infection, and to determine the persistence of antibodies across time.

Acknowledgements The authors thank all the AVIS-Milano volunteers for their support with blood collection, Patrizia Angiolillo for electronic questionnaire preparation, Nicla Diomede for informatics security and all the University of Milan staff and volunteers for their precious help.

Collaborators The UNICORN Consortium investigators: Claudio Bandi (Department of Biosciences and Pediatric Clinical Research Center 'Romeo and Enrica Invernizzi', University of Milan, Milan, Italy), Tommaso Bellini (Department of Medical Biotechnology and Translational Medicine, University of Milan, Milan, Italy), Matteo Bonzini (EPIGET Lab, Department of Clinical Sciences and Community Health, Università degli Studi di Milano, Milan, Italy; Occupational Health Unit, Fondazione IRCCS Ca' Granda Ospedale Maggiore Policlinico, Milan, Italy), Marco Buscaglia (Department of Medical Biotechnology and Translational Medicine, University of Milan, Milan, Italy), Carlo Cantarella (EPIGET Lab, Department of Clinical Sciences and Community Health, Università degli Studi di Milano, Milan, Italy), Laura Cantone (EPIGET Lab, Department of Clinical Sciences and Community Health, Università 
degli Studi di Milano, Milan, Italy), Michele Carugno (EPIGET Lab, Department of Clinical Sciences and Community Health, Università degli Studi di Milano, Milan, Italy; Occupational Health Unit, Fondazione IRCCS Ca' Granda Ospedale Maggiore Policlinico, Milan, Italy), Sergio Casartelli (Associazione Volontari Italiani Sangue (AVIS) Milano, Milan, Italy), Sarah D'Alessandro (Department of Biomedical, Surgical and Dental Sciences, Laboratory of Translational Research, Via Carlo Pascal 36, Milano, Italy), Francesca De Chiara (AVIS Milano, Milan, Italy), Serena Delbue (Department of Biomedical, Surgical and Dental Sciences, Laboratory of Translational Research, Via Carlo Pascal 36, Milano, Italy), Ivano Eberini (Department of Pharmacological and Biomolecular Sciences, University of Milan, Milan, Italy), Luca Ferrari (EPIGET Lab, Department of Clinical Sciences and Community Health, Università degli Studi di Milano, Milan, Italy), Monica Ferraroni (Branch of Medical Statistics, Biometry, and Epidemiology 'G A Maccacaro', Department of Clinical Sciences and Community Health, Università degli Studi di Milano, Milan, Italy), Laura Galastri (AVIS Milano, Milan, Italy), Cristina Galli (Department of Biomedical Sciences for Health, University of Milan, Milan, Italy), Simona lodice (EPIGET Lab, Department of Clinical Sciences and Community Health, Università degli Studi di Milano, Milan, Italy), Carlo La Vecchia (Branch of Medical Statistics, Biometry, and Epidemiology 'G A Maccacaro', Department of Clinical Sciences and Community Health, Università degli Studi di Milano, Milan, Italy), Chiara Macchi (Department of Pharmacological and Biomolecular Sciences, University of Milan, Milan, Italy), Ilaria Manini (Department of Biomedical, Surgical and Dental Sciences, Laboratory of Translational Research, Via Carlo Pascal 36, Milano, Italy), Serena Marchi (Department of Biomedical, Surgical and Dental Sciences, Laboratory of Translational Research, Via Carlo Pascal 36, Milano, Italy), Jacopo Mariani (EPIGET Lab, Department of Clinical Sciences and Community Health, Università degli Studi di Milano, Milan, Italy), Massimiliano Ruscica (Department of Pharmacological and Biomolecular Sciences, University of Milan, Milan, Italy), Tommaso Schioppo (Division of Rheumatology, ASST Pini-CTO, Milan, Italy), Letizia Tarantini (EPIGET Lab, Department of Clinical Sciences and Community Health, Università degli Studi di Milano, Milan, Italy), Claudia Maria Trombetta (Department of Molecular and Developmental Medicine, University of Siena, Siena, Italy), Marco Vicenzi (Fondazione IRCCS Ca' Granda Ospedale Maggiore Policlinico, Cardiovascular Disease Unit, Internal Medicine Department, Milan, Italy; Dyspnea Lab, Department of Clinical Sciences and Community Health, University of Milan, Milan, Italy), Giuliano Zanchetta (Department of Medical Biotechnology and Translational Medicine, University of Milan, Milan, Italy).

Contributors GPM: conceptualisation, funding acquisition, writing-original draft. FR: investigation, writing-original draft. CF: data curation, formal analysis. LD, AM, MH, BA: methodology. EP: supervision, writing-review. ACP: writing-review. EM: conceptualisation, supervision. VB: conceptualisation, funding acquisition, writingreview. UNICORN Consortium investigators: supervision.

Funding This work was supported by the Funding Action 'Ricerche Emergenza coronavirus', University of Milan, 2020. The authors acknowledge support from the University of Milan through the APC initiative.

Competing interests None declared.

Patient and public involvement Patients and/or the public were involved in the design, or conduct, or reporting, or dissemination plans of this research. Refer to the Methods section for further details.

Patient consent for publication Not required.

Ethics approval The study was approved by the Ethics Committee of the University of Milan (approval number 17/20, approval date 6 March 2020) and was conducted in accordance with the Declaration of Helsinki. All participants signed an informed consent form.

Provenance and peer review Not commissioned; externally peer reviewed.

Data availability statement All data relevant to the study are included in the article or uploaded as supplementary information. $n / a$.

Open access This is an open access article distributed in accordance with the Creative Commons Attribution Non Commercial (CC BY-NC 4.0) license, which permits others to distribute, remix, adapt, build upon this work non-commercially, and license their derivative works on different terms, provided the original work is properly cited, appropriate credit is given, any changes made indicated, and the use is non-commercial. See: http://creativecommons.org/licenses/by-nc/4.0/.

ORCID iD

Valentina Bollati http://orcid.org/0000-0002-0370-9598
REFERENCES

1 Spiteri G, Fielding J, Diercke M, et al. First cases of coronavirus disease 2019 (COVID-19) in the WHO European region, 24 January to 21 February 2020. Euro Surveill 2020;25.

2 Grasselli G, Pesenti A, Cecconi M. Critical care utilization for the COVID-19 outbreak in Lombardy, Italy: early experience and forecast during an emergency response. JAMA 2020;323:1545-6.

3 Indolfi C, Spaccarotella C. The outbreak of COVID-19 in Italy: fighting the pandemic. JACC Case Rep 2020;2:1414-8.

$4 \mathrm{Li} \mathrm{C}$, Romagnani P, von Brunn A, et al. SARS-CoV-2 and Europe: timing of containment measures for outbreak control. Infection 2020;48:483-6.

5 Oran DP, Topol EJ. Prevalence of asymptomatic SARS-CoV-2 infection : a narrative review. Ann Intern Med 2020;173:362-7.

6 Feng W, Zong W, Wang F, et al. Severe acute respiratory syndrome coronavirus 2 (SARS-CoV-2): a review. Mol Cancer 2020;19:100.

7 Kronbichler A, Kresse D, Yoon S, et al. Asymptomatic patients as a source of COVID-19 infections: a systematic review and metaanalysis. Int $J$ Infect Dis 2020;98:180-6.

8 Dioni L, Albetti B, Rota F. SARS-CoV-2 RNA detection in nasal midturbinate swabs. Research Square 2020.

9 Lai X, Wang X, Yang Q, et al. Will healthcare workers improve infection prevention and control behaviors as COVID-19 risk emerges and increases, in China? Antimicrob Resist Infect Control 2020;9:83.

10 Mazzini L, Martinuzzi D, Hyseni I, et al. Comparative analyses of SARS-CoV-2 binding ( $\lg G, \lg M, \lg A)$ and neutralizing antibodies from human serum samples. $J$ Immunol Methods 2021;489:112937.

11 Alfano V, Ercolano S. The efficacy of lockdown against COVID-19: a cross-country panel analysis. Appl Health Econ Health Policy 2020;18:509-17.

12 Ghosal S, Bhattacharyya R, Majumder M. Impact of complete lockdown on total infection and death rates: a hierarchical cluster analysis. Diabetes Metab Syndr 2020;14:707-11.

13 Alicandro G, Remuzzi G, La Vecchia C. Italy's first wave of the COVID-19 pandemic has ended: no excess mortality in May, 2020. Lancet 2020;396:e27-8.

14 Espinoza B, Castillo-Chavez C, Perrings C. Mobility restrictions for the control of epidemics: when do they work? PLoS One 2020;15:e0235731

15 Lowen AC, Mubareka S, Steel J, et al. Influenza virus transmission is dependent on relative humidity and temperature. PLoS Pathog 2007;3:e151-6.

16 Domingo JL, Rovira J. Effects of air pollutants on the transmission and severity of respiratory viral infections. Environ Res 2020;187:109650.

17 Man WH, de Steenhuijsen Piters WAA, Bogaert D. The microbiota of the respiratory tract: gatekeeper to respiratory health. Nat Rev Microbiol 2017;15:259-70

18 Gandhi M, Yokoe DS, Havlir DV. Asymptomatic transmission, the achilles' heel of current strategies to control Covid-19. N Engl J Med 2020;382:2158-60.

19 Shields A, Faustini SE, Perez-Toledo M, et al. SARS-CoV-2 seroprevalence and asymptomatic viral carriage in healthcare workers: a cross-sectional study. Thorax 2020;75:1089-94.

20 Perico L, Tomasoni S, Peracchi T, et al. COVID-19 and lombardy: TESTing the impact of the first wave of the pandemic. EBioMedicine 2020;61:103069.

21 Long Q-X, Tang X-J, Shi Q-L, et al. Clinical and immunological assessment of asymptomatic SARS-CoV-2 infections. Nat Med 2020;26:1200-4.

22 Milani GP, Dioni L, Favero C, et al. Serological follow-up of SARSCoV-2 asymptomatic subjects. Sci Rep 2020;10:20048.

23 Duan S, Zhou M, Zhang W, et al. Seroprevalence and asymptomatic carrier status of SARS-CoV-2 in Wuhan City and other places of China. PLoS Negl Trop Dis 2021;15:e0008975.

24 Grant MC, Geoghegan L, Arbyn M, et al. The prevalence of symptoms in 24,410 adults infected by the novel coronavirus (SARSCoV-2; COVID-19): a systematic review and meta-analysis of 148 studies from 9 countries. PLoS One 2020;15:e234765.

25 Tu Y-P, Jennings R, Hart B, et al. Swabs collected by patients or health care workers for SARS-CoV-2 testing. N Engl J Med 2020;383:494-6.

26 Guo L, Ren L, Yang S, et al. Profiling early humoral response to diagnose novel coronavirus disease (COVID-19). Clin Infect Dis 2020;71:778-85. 